\title{
The paraneoplastic meaning of R3SPE (remitting seronegative simmetrical synovitis with pitting edema) syndrome.
}

\author{
Ciro Manzo \\ Internal and Geriatric Department, Chief of Geronthorheumatological, Outpatient Clinic, "Mariano Lauro" hospital, \\ Sant'Agnello, Naples, Italy.
}

\begin{abstract}
Introduction: Remitting seronegative symmetrical synovitis with pitting edema (RS3PE) is an uncommon elderly-onset rheumatic disease characterized by tenosynovitis of both flexor and extensors tendons at the wrist and at the feet. Cancer has been reported in association with this syndrome since 1985 .

Materials and method: We perform a systematic electronic search of Medline and PubMed for evaluation of relationship between cancer and RS3PE and present in this review the more significant data.
\end{abstract}

Results: The paraneoplastic weight of RS3PE syndrome is greater than other clinical pictures. In our experience, when RS3PE is associated with polymyalgia rheumatic (PMR), the probability of a paraneoplastic picture is very high $(28.5 \%$ in our PMR+RS3PE cohort had a cancer diagnosed in an observation time of 24 months vs. $2.04 \%$ in cohort of PMR without RS3PE).

Conclusion: The possibility that RS3PE can be considered a paraneoplastic phenomenon or a generic risk factor for cancer is still debated. The repercussions of these evaluations on health policies are easily understandable. More data must be available.

Keywords: RS3PE syndrome, Paraneoplastic syndromes, Elderly, Vascular endothelial growth factor.

Accepted October 13, 2016

\section{Introduction}

Remitting Seronegative Symmetrical Synovitis with Pitting Edema (RS3PE) is an uncommon elderly-onset rheumatic picture descripted for the first time by McCarthy et al. in 1985 [1] characterized by tenosynovitis of extensor tendons at the wrist and (less frequently) at the feet. The edematous imbibition of tendon sheaths determines the "boxing-glove" swelling which can be considered - when bilateral-virtually pathognomonic (Figure 1).

Like the eponym highlighted, RS3PE syndrome is bilateral, symmetric, and seronegative for rheumatoid factor and antibodies against citrullinated peptides. The only case in which the clinical manifestations are unilateral or asymmetric regards the limb paretic [2,3]. In the descriptions by McCarthy et al. the association between this clinical picture with HLA B7 subtype was very strong (six of eight patients in the first paper; seven out of 13 in the second paper) $[1,4]$. Histocompatibility Lymphocytic Antigen (HLA) B7 subtype is part of the HLA complex related to B27. The seronegative spondyloarthritis (SSA),

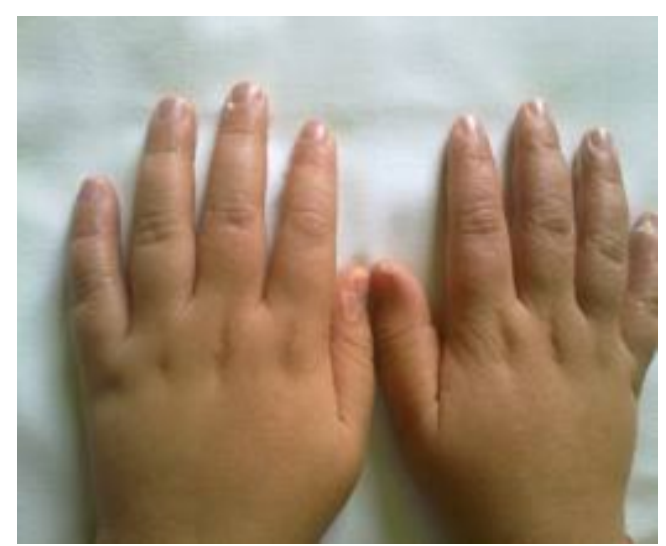

Figure 1. RS3PE syndrome with "boxing-glove” swelling

often associated with HLA-B27 and related alleles, are the diseases in which the syndrome frequently manifests itself. Its clinical picture characteristically responds to low-dosages corticosteroids (5-10 mg prednisone or equivalent/day). Its removal occurs rapidly (few days) even if the duration of corticosteroid therapy is longer 
(several months, as average). Its relapse is extremely rare in the "benign" forms and, when present, it presents only after several months [5].

\section{Rs3pe Syndrome in the Clinical Practice}

RS3PE can represent a manifestation of some diseases, especially rheumatic inflammatory diseases. Among these, polymyalgia rheumatica (PMR), rheumatoid arthritis (RA) and SSA are the most frequent [6]. It is estimated that no more than $10 \%$ of patients with PMR may have a RS3PE syndrome and some authors think that RS3PE can be considered an integral part of the spectrum of the PMR manifestations [7]. Nevertheless, some differences have been highlighted between PMR and RS3PE [5,8]. Anedoctal is the appearance of this syndrome in the course of Parkinson's disease or after a cerebral hemorrhage $[9,10]$. Besides, RS3PE has been associated with drugs.

For example, Yamauchi et al. described 2 cases of RS3PE associated with treatment with a dipeptidyl peptidase-4 (DPP4) inhibitor [11]. A case of this syndrome due to insulin therapy has been also descripted [12] as well as a case associated with rifampicin [13]. The possibility that RS3PE can be a disease (and not a syndrome) has been more and more evaluated : this possibility was basically excluced [14-17].

\section{Rs3pe Syndrome as Paraneoplastic Phenomenon}

Since 1985, tumors have been descripted in association with RS3PE [1]. Over the years, several types of tumors have been described associated with the syndrome and its paraneoplastic warning has been again and again highlighted [18-22]. The possibility that the syndrome may be the initial clinical manifestation for cancer diagnosed more than 10 years later does not easy to share [23]. In fact, there is a difference between RS3PE as paraneoplastic phenomenon and its association (in a casual way) with a cancer. As well-known, the term "paraneoplastic syndromes (PS)" includes all the various symptoms not attributable to direct tumor invasion or compression. A period between appearance of RS3PE and cancer greater than two years can exclude the diagnosis of PS sensu strictiori [24-26]. The levels of vascular endothelial growth factor (VEGF)-a cytokine able to increase vascular permeability and dilation-are significantly higher in RS3PE patients than in controls and its levels decreased after glucocorticoid treatment [25].

Both synovial hypervascularity (synovitis) and increment of vascular permeability (subcutaneous oedema) may be facilitated by VEGF in patients with RS3PE syndrome. The importance of VEGF in the neoplastic spreading is well-known [26] but the real importance of VEGF in the paraneoplastic potentiality of RS3PE remains speculative until today. It is estimated that paraneoplastic syndromes affect up to $8 \%$ of patients with cancer [24] but in elderly patients with rheumatic diseases RS3PE can represent a neoplastic marker up to $20 \%$ of cases [5]. The greatest percentages observed in patients with RS3PE syndrome warrant attention in paraneoplastic direction. This attention is particularly suitable in elderly patients where RS3PE is associated with PMR.

We have evaluated 200 elderly patients ( $>65$ years old) with PMR consecutively observed at our rheumatologic outpatient clinic. The diagnosis of PMR was made until 2013 - using the criteria proposed by Chuang [27] and after 2013 using the criteria proposed by the European League against Rheumatism (EULAR) and the American College of Rheumatology (ACR) [28]. The observation time for the appearance of cancer was of 24 months from initial diagnosis of PMR. In the same cohort of patients, was highlighted the presence of RS3PE syndrome in a binary way (yes/no): The fact that the RS3PE is the first clinical manifestation or not did not constitute an element of assessment. The presence of RS3PE was observed in only 7 patients with PMR and in three of these was possible to recognize a tumor: prostatic cancer, vesical cancer, multiple myeloma.

Table 1 shows the baseline characteristics of these 3 patients. In all these patients, RS3PE presented before the discovery of the malignancies; the treatment of neoplasias determined the total and permanent disappearance of RS3PE syndrome; no recurrence was observed during follow-up. When the two groups (PMR without RS3PE vs. PMR+RS3PE) were compared, the disease duration of PMR before the diagnosis of cancer was in the first group double that in the second group [29] (Table 2). In 193 PMR without RS3PE patients, only in two cases PMR represented a paraneoplastic manifestation: in the first case of a neuroendocrine tumor gastric gastrin-secreting; in the second case of a non-Hodgkin lymphoma. In the first case, (man 67 years old) a concomitant macrocytic anemia was the key to reaching this final diagnosis [30]. In a recent meta-analysis of 331 cases of RS3PE, malignancy was reported in 54 cases $(16.31 \%)$ but a concurrent rheumatologic condition was reported only in 22 cases $(6.65 \%)$ and a concurrent PMR only in anecdotal cases [31]. This percentage is not much different from the average malignancy rate estimated to be $20 \%$ [5]. However, very low percentage of patients with PMR and RS3PE syndrome did not give a meaningful contribution about the paraneoplastic weight of the association RS3PE+PMR.

Table 1. Baseline characteristics of the three patients with $P M R+R S 3 P E+$ Cancer

Gender, n (\%): Men 2 (66.6\%) - Woman 1 (33.3\%)

Age at the date of examination - median (min-max): 72 years (66-79)

ESR - median (min-max) $[\mathrm{mm} / \mathrm{h}]=110(100-115)$

$\mathrm{CRP}-$ median (min-max) $[\mathrm{mg} / \mathrm{L}]=42.0(25.9-66.6)$

Disease duration before the diagnosis of cancer - median

(min-max) [month]: 8 (3-10)

Note: ESR: Erythrocyte sedimentation rate; CRP: C-Reactive Protein 
Table 2: Differences between $P M R / C a n c e r+R S 3 P E$ vs. PMR + Cancer without $R S 3 P E$

\begin{tabular}{|l|c|c|}
\hline \multicolumn{1}{|c|}{ PMR+RS3PE: n. 3/7 } & PMR-RS3PE=n. 2/193 \\
\hline Gender, $\mathrm{n}(\%)$ & Men, 2 (66.6\%) & Men, 63 (32.1\%) \\
\hline Gender, $\mathrm{n}(\%)$ & Female, 1 (33.3\%) & Female, 130 $(67,9 \%)$ \\
\hline Age at the date of examination, median (min-max) & 72 years (66-79) & years $(65-91)$ \\
\hline ESR - median (min-max) $[\mathrm{mm}(\mathrm{h}]$ & $110(100-115)$ & $70(30-88)$ \\
\hline CRP - median (min-max) $[\mathrm{mg} / \mathrm{L}]$ & $42.0(25.9-66.6)$ & $16(12-10)$ \\
\hline $\begin{array}{l}\text { Disease duration before the diagnosis } \\
\text { of cancer, median (min-max) [months }]\end{array}$ & $8(3-10)$ & \\
\hline
\end{tabular}

Note: ESR: Erythrocyte sedimentation rate; CRP: C-Reactive Protein

Table 3. Minimum diagnostic set in absence of organ-specific signs or symptoms, useful in patients with RS3PE suspected for a paraneoplastic syndrome

Complete blood count

Protein electrophoresis framework

Searching for occult blood in the stool

Total and free prostatic specific antigen (men, only)

Abdominal, pelvic and lymph nodes ultrasonography

Breast ultrasound (female, only)

Thin layer CT chest

In our experience, RS3PE is rarely associated with PMR (3.5\% in the presented data) but its presence in patients with PMR is associated with a very high risk for cancer in the first 24 months of observation $(28.5 \%$ PMR+RS3PE vs. only $2.04 \%$ PMR without RS3PE). The relationship between PMR alone and cancer was also evaluated by our group [32-35]. As in the experience of other groups, the lack of response to corticosteroid therapy and a rapid reappearance of RS3PE after the end of this therapy have to be considered the most important warning in paraneoplastic direction. However, in the clinical practice some patients can have no or a modest response to a glucocorticoid and a good response using a different glucocorticoid in an equivalent dosage [34] and this possibility must be consider before to diagnose a lack of response to glucocorticoid therapy. In our experience, the presence of important systemic manifestation is less frequent $[35,36]$. In absence of specific signs or symptoms, a minimum diagnostic set is necessary if we suspect a paraneoplastic nature of RS3PE (Table 3). Instead in presence of specific signs or symptoms, the diagnostic steps will be guided by them.

\section{Conclusion}

RS3PE syndrome can represent a paraneoplastic phenomenon. Its paraneoplastic weight is greater than other syndromes. In our experience, when RS3PE is associated with PMR (the more common inflammatory rheumatic disease in elderly over 70 years old), the probability of a paraneoplastic picture is very high. The lack of response to glucocorticoid therapy and the prompt relapse of RS3PE after the discontinuation of glucocorticoid therapy are the most important warnings. In presence of specific signs and/or symptoms, the diagnostic steps will be different according to these. If these are absent, a minimum diagnostic set is necessary in all patients. The onset of cancer several years after RS3PE could be represent an occasional association instead of a casual relationship. Regarding this point, studies from multicenter cohorts are necessary and more accurate data should be made avaliable. The repercussions on health policies of cancer risk in elderly with RS3PE are easily understandable and therefore the detection of all potential bias is mandatory.

\section{Acknowledgement}

The author thanks Maria Natale MD, Raffaele Cioffi MD, Teresa Puca, registered nurse for their collaboration in the rheumatologic outpatient clinic of "Mariano Lauro" hospital. This review is dedicated to the general practitioners of the Sorrento peninsula and Amalfi coast.

\section{References}

1. McCarty DJ, O' Duffy JD, Pearson L, et al. Remitting seronegative symmetrical synovitis with pitting edema: RS3PE syndrome. JAMA 1985; 254: 2763.

2. Pariser KM, Canoso JJ. Remitting, seronegative (A) symmetrical synovitis with pitting edema--two cases of RS3PE syndrome. J Rheumatol 1991; 18: 1260-1262.

3. Keenan RT, Hamalian GM, Pillimger MH. RS3PE presenting in a unilateral pattern: Case report and review of the literature. Semin Artrihtis Rheum 2009; 38: 428-433.

4. Russell EB, Hunter JB, Pearson L, et al. Remitting, seronegative, symmetrical synovitis with pitting edema-13 additional cases. J7 Rheumatol 1990; 17: 633-639.

5. Li H, Altman RD, Yao Q. RS3PE: Clinical and research development. Curr Rheumatol Rep 2015; 17: 49.

6. Manzo C, Stisi S. RS3PE syndrome: Three messages useful for the geriatrician. Ger Extraosp 2016.

7. Salvarani C, Gabriel S, Hunder GG. Distal extremity swelling with pitting edema in polymyalgia rheumatica: Report of nineteen cases. Arthritis Rheum 1996; 39: 73-80.

8. Kimura $\mathrm{M}$, Tokuda $\mathrm{Y}$, Oshiawa $\mathrm{H}$, et al. Clinical characteristics of patients with remitting seronegative symmetrical synovitis with pitting edema compared to patients with pure polymyalgia rheumatic. J Rheumatol 2012; 39: 148-153.

9. Ito E, Okamoto H, Mochizuki A, et al. Remitting seronegative symmetrical synovitis with pitting edema (RS3PE) syndrome accompanied by Parkinson's disease.

10. Yang DH, Chang DM, Lai JH et al. Remitting seronegative symmetrical synovitis with pitting edema following acute intracranial hemorrhage. Rheumatol Int 2011; 31: 101-104. 
11. Yamauchi K, Sato Y, Yamashita K et al. RS3PE in association with dipeptidyl peptidase-4 inhibitor: report of two cases. Diabetes Care 2012; 35: e7.

12. Mainali NR, Schmidt TR, Alweis R. Novel development of remitting seronegative symmetrical synovitis with pitting edema (RS3PE) syndrome due to insulin therapy. Am J Case Rep 2014; 15: 119-122.

13. Smyth D, Rehman R, Remund $\mathrm{K}$, et al. Remitting seronegative symmetrical synovitis with pitting oedema associated with rifampicin. Ir J Med Sci 2011; 180: 585-586.

14. Yao Q, Su X, Altman RD. Is remitting seronegative symmetrical synivitis with pitting edema (RS3PE) a subset of rheumatoid arthritis? Semin Arthritis Rheum 2010; 40: 89-94.

15. Queiro R. RS3PE syndrome: A clinical and immunogenetical study. Rheumatol Int 2004; 24: 103-105.

16. Schaeverbeke T, Vernhes JP, Bannwarth B, et al. Is remitting seronegative symmetrical synovitis with pitting oedema (RS3PE) syndrome associated with HLA-A2 ? Br J Rheumatol 1995; 34 : 889-890.

17. Cantini F, Salvarani C, Olivieri et al. Remitting seronegative symmetrical synovitis with pitting oedema (RS3PE) syndrome: A prospective follow-up and magnetic resonance imaging study. Ann Rheum Dis 1999; 58: 230-236.

18. Sibilia J, Friess S, Schaeverbeke $\mathrm{T}$ et al. Remitting seronegative symmetrical synovitis with pitting edema (RS3PE): A form of paraneoplastic polyarthritis? J Rheumatol 1999; 26: 115-120.

19. Cantini F, Salvarani C, Olivieri I. Paraneoplastic remitting seronegative symmetrical synovitis with pitting edema. Clin Exp Rheumatol 1999; 17: 741.

20. Bruscas Izu C, Puertas JR, Cabrerizo-Garcia JL. RS3PE syndrome as a first manifestation of bladder carcinoma. Reumatol Clin 2013; 9: 74

21. Ferrao C, Faria RM, Farrajota P, et al. Lucky to meet RS3PE. J Rheumatol 2005; 32:1760-1761.

22. Emamifar A, Hess S, Gildberg-Mortensen R, et al. Association of remitting seronegative symmetrical synovitis with pitting edema, polymyalgia rheumatica and adenocarcinoma of the prostate. Am J Case Rep 2016; 17: 60-64.

23. Russell EB. Remitting seronegative symmetrical synovitis with pitting edema syndrome: Follow-up for neoplasia. J Rheumatol 2005; 32: 1760-1761.

24. Manger B, Schett G. Paraneoplastic syndromes in rheumatology. Nat Rev Rheumatol 2014; 10: 662-670.

25. Arima K, Origuchi T, Tamai M, et al. RS3PE syndrome presenting as vascular endothelial growth factor associated disorder. Ann Rheum Dis 2005; 64: 1653-1655.

26. Mittal K, Ebos J, Rini B. Angiogenesis and the tumor microenvironment: Vascular endothelial growth factor and beyond. Semin Oncol 2014; 41: 235-251.

27. Marie I. Giant cell arteritis and polymyalgia rheumatica: Diagnostic criteria. Rev Med Interne 2013; 34: 403-411.

28. Dasgupta B, Cimmino MA, Maradit-Kremers H, et al. 2012 provisional classification criteria for polymyalgia rheumatica. A European League against Rheumatism/ American College of Rheumatology Collaborative Initiative. Ann Rheum Dis 2012; 71: 484-492.

29. Manzo C, Natale M. Association of remitting seronegative symmetrical synovitis with pitting edema (rs3pe syndrome) and polymyalgia rheumatica: A neoplastic warning? Geriatrics 2016.

30. Manzo C. About a gastric neuroendocrine tumor presenting as polymyalgia rheumatica and macrocytic anemia. J Clin Case Rep 2016; 6: 5.

31. Kaemacharva P, Donato AA, Aryal MR, et al. RS3PE revisited: A systematic review and meta-analysis of 331 cases. Clin Exp Rheumatol 2016.

32. Manzo C. Cancerogenesis and polymyalgia rheumatica. J Carcinog Mutagen 2016.

33. Manzo C, Natale M. Polymyalgia rheumatica and cancer risk: The importance of the diagnostic set. Open Access Rheumatology: Review and Research 2016.

34. Manzo C. La polimialgia reumatica: Cosa è utile sapere per il geriatra territoriale? Ger Extraosp 2009; 7: 9-11.

35. Manzo C, Natale M, Cappiello F. Quanti anziani con Polimialgia Reumatica passano sotto gli occhi del medico del territorio ? Ger Extraosp 2008; 6: 12-15.

36. Pelosof LC, Gerber DE. Paraneoplastic syndromes: An approach to diagnosis and treatment. Mayo Clin Proc 2010; 85: 838-854

\section{Correspondence to:}

Dr. Ciro Manzo,

Internal and Geriatric Department,

Chief of Geronthorheumatological,

Outpatient Clinic, "Mariano Lauro" Hospital,

Sant'Agnello,

Naples,

Italy.

Tel: $+390815331465 ; 3389436790$

E-mail: cirmanzo@libero.it 